\title{
TRATAMENTO FARMACOLÓGICO DA HIPERTENSÃO ARTERIAL ESSENCIAL
}

\author{
PHARMACOLOGY TREATMENT OF ARTERIAL HYPERTENSION
}

Wille Oigman

Docente da Clínica Médica UERJ

CORRESPONDÊNCIA: Rua Barão da Torre, 295/402 - CEP: 22411-001 - Rio de Janeiro - RJ

OIGMAN W. Tratamento farmacológico da hipertensão arterial essencial. Medicina, Ribeirão Preto, 29: 244-249, abr./set. 1996.

RESUMO: O artigo avalia de maneira objetiva as 7 principais famílias de anti-hipertensivos, em uso corrente na prática médica. Procura-se enfatizar, fundamentalmente, suas vantagens e desvantagens. O autor procura posicionar cada família de anti-hipertensivos, fortalecendo a individualização do tratamento, além de acentuar as indicações específicas quando da presença de outras doenças ou de outros fatores de riscos para doença cardiovascular. Procura-se, também, chamar a atenção para as repercussões hemodinâmica e metabólicas que cada anti-hipertensivo poderá determinar.

UNITERMOS: Anti-hipertensivos. Hipertensão; farmacologia.

O tratamento da hipertensão arterial humana (HA) vem, nos últimos anos, saindo progressivamente do empirismo - ensaio de acertos e erros - em direção a drogas que levam a bloqueios ou antagonismos mais específicos.

Desde o início dos anos 80, os estudos epidemiológicos vêm demonstrando, claramente, uma expressiva redução dos eventos cardiovasculares. Contudo, este fato tem sido observado principalmente no acidente vascular cerebral, enquanto menos expressivos têm sido os efeitos sobre a insuficiência coronariana e seus complicadores - infarto do miocárdio e morte súbita.

Não faz parte desta revisão uma discussão mais profunda das razões que possam explicar tais diferenças. Contudo, seria importante mencionar as principais causas envolvidas e, entre elas citaríamos: as alterações metabólicas (aceleração da ateroesclerose) e eletrolíticas (arritmias e morte súbita), promovidas por doses elevadas e a longo prazo dos diuréticos e beta bloqueadores, além de prováveis alterações hemodinâmicas, com reduções excessivas da pressão arterial que pudesse comprometer a reserva coronariana (curva $\mathbf{J}$ ).

Nos dias atuais, 1996, poderíamos dividir em 7 distintos grupos ou famílias as drogas para o tratamento da Hipertensão Arterial, a saber :

1. Diuréticos

2. Simpaticolíticos de ação central

3. Beta bloqueadores

4. Alfa bloqueadores

5. Antagonistas de canais de cálcio

6. Inibidores da Enzima Conversora

7. Antagonistas de receptor da Angiotensina II 


\section{EFICÁCIA TERAPÊUTICA}

Os diuréticos tiazídicos e a clortalidona apresentam a maior eficácia terapêutica, controlam a pressão arterial em cerca de 60 a $70 \%$ dos hipertensos leves. Os beta bloqueadores vêm logo a seguir apresentando, também, uma boa eficácia terapêutica. As demais drogas anti-hipertensivas apresentam um perfil semelhante entre si, contudo inferior aos diuréticos, quando empregadas como monoterapia para hipertensão leve.

Quando consideramos hipertensos mais graves, considerando-se apenas o número, em geral, tem-se preferido a associação de drogas ao invés, de se aumentar progressivamente a dose do anti-hipertensivo. Há evidências de que assim se procedendo estaríamos, de maneira significativa, reduzindo os efeitos colaterais decorrentes das doses elevadas de qualquer anti-hipertensivo.

\section{VANTAGENS E DESVANTAGENS DOS AGEN- TES ANTI-HIPERTENSIVOS}

\subsection{Diuréticos}

Os diuréticos apresentam vantagens importantes: podem ser administrados uma vez ao dia, fator decisivo em aumentar a aderência ao tratamento, raramente desenvolvem tolerância e são de custo bem inferior aos demais anti-hipertensivos.

Com o uso prolongado e em doses elevadas- acima de $25 \mathrm{mg} /$ dia - podem levar a alterações hidroeletrolíticas e metabólicas de relevância clínica. É comum observarmos, na prática diária, que pacientes obesos ou que já apresentam níveis de glicemia discretamente elevados, passarem a apresentar níveis plasmáticos de glicose nos níveis patológicos. Esse fato deve-se, provavelmente, a um aumento na resistência periférica de insulina, acentuada pela hipocalemia associada.

Das alterações hidroeletrolíticas, a hipocalemia é a mais freqüente. Não ocorre tão precocemente quanto se esperava mas, quando presente, significa uma espoliação intracelular marcante, podendo determinar complicações graves como arritmias complexas, principalmente na presença de hipertrofia ventricular esquerda. A hipomagnesemia está, também, presente associada à hipocalemia severa, devendo-se nesta situação repor ambos os íons.

Quanto ao ácido úrico é comum observarmos elevações séricas, por vezes, substanciais. Contudo, não é freqüente encontrarmos quadro agudo de gota. A elevação nos níveis plasmáticos de colesterol é outro distúrbio metabólico descrito no uso prolongado com diuréticos. Apesar de atingir significância estatística quando analisada ao nível de grandes populações e quando avaliada ao nível individual, essas elevações pouco freqüentemente necessitam de tratamento específico.

Os diuréticos apresentam um efeito muito discreto na reversão da hipertrofia ventricular esquerda. Em pacientes idosos e naqueles com algum grau de insuficiência renal, os diuréticos tiazídicos podem deteriorar a função renal, diminuem o fluxo plasmático renal e podem elevar os níveis séricos de uréia e creatinina.

\subsection{Simpaticolíticos de ação central}

Tanto a metildopa quanto a clonidina apresentam poucas contra-indicações ao seu uso em hipertensos. Contudo, como são drogas de ação pouco específica determinam freqüentemente efeitos colaterais intoleráveis, p.ex., secura na boca, sonolência, hipotensdiminuição no libido.

São eficazes como monoterapia porém, determinam um tolerância precoce necessitando associação de outra droga, em geral um diurético, para manutenção do seu efeito hipotensor.

A metildopa apresentam um efeito expressivo na reversão da hipertrofia ventricular esquerda e não deteriora a função renal. Por vezes pode determinar o aparecimento de teste de Coombs positivo, associado com anemia hemolítica.

Pelo seu lado a clonidina tem a grande vantagem de ser de baixo custo; contudo, sua grande desvantagem esta no fato de que a sua suspensão muito abrupta pode levar à crise hipertensiva.

\subsection{Beta bloqueadores}

Os betas bloqueadores apresentam um perfil hipotensor muito próximo aos diuréticos quando administrado como monoterapia. Apresentam algumas vantagens importantes como da prevenção do secundária do infarto do miocárdio, em pacientes com síndrome anginosa associada, em hipertensos hipercinéticos e, também, naqueles com enxaqueca ou com tremor intencional.

Dentre suas desvantagens, ressaltam-se: apresenta um perfil hemodinâmico indesejável por deixar livre os receptores alfa que são vasoconstrictores, determinando alterações clínicas como mãos frias. 
Tem contra-indicação formal em pacientes asmáticos ou com doença pulmonar obstrutiva crônica, com doença vascular arterial periférica, com insuficiência cardíaca principalmente do tipo sistólica.

Sob ponto de vista metabólico podem desenvolver elevações expressivas nos níveis séricos dos triglicerídeos, além de reduzirem a resposta simpática de alerta em diabéticos, quando desenvolvem hipoglicemia severa e sintomática.

\subsection{Alfa bloqueadores}

Os alfa bloqueadores apresentam um bom perfil hemodinâmico reduzindo, significativamente, a resistência vascular periférica. Contudo, por razões desconhecidas, desenvolvem tolerância muito rápida o que leva progressivamente à necessidade em se aumentar a dose do medicamento para se atingir o mesmo efeito hipotensor e, com isso, determinam aumento expressivo nos efeitos colaterais e no custo do tratamento.

Apresentam como vantagem não induzirem alterações metabólicas, podendo até reduzir discretamente os níveis séricos de colesterol.

\subsection{Antagonistas de canais de cálcio.}

Os antagonistas de canais de cálcio (ACC) são um grupo, bioquimicamente, heterogêneo de drogas que interferem com a entrada de cálcio dentro da célula, e com isso, interferindo com as funções intracelular do cálcio tanto nos seus efeitos constrictores quanto nos efeitos tróficos.

Há 4 grupos distintos de ACC: Os derivados dihidropiridínicos (nifedipina, amlodipina, isradipina, felodipina, lacidipina); os derivados feniaalkilaminas (verapamil), os do grupo dos benzotiapínicos (diltiazem) e mais recentemente um novo grupo representado pelo mebefradil cujo efeito distinto está em antagonizar os canais $\mathrm{T}$, e dessa maneira, apresenta efeitos mistos: cardiotropismo negativo sem desenvolver redução no inotropismo e redução na resistência vascular periférica.

Os ACC apresentam um expressivo efeito hipotensor como monoterapia em hipertensos leves e moderados, constituindo-se, nos dias atuais, como uma das opções iniciais para o tratamento dos hipertensos. Apesar de alguns considerarem os ACC mais indicados para indivíduos idosos e /ou com atividade plasmática de renina reduzida ( $\mathrm{p}$.ex. negros) os resultados obtidos na prática diária não sustentam estas afirmações sendo eficazes independentes da idade ou da APR.
Recentemente, temos apresentações que levam a uma vida média mais prolongada como é caso da amlodipina, lacidipina e mebefradil, ou formulações galênicas de liberação em 24 horas como a nifedipina OROS, a felodipina retard, entre outras. Estas formulações aumentam a relação peak-trough (vale-pico) que, na prática, têm o significado de poderem ser administradas uma vez ao dia, aumentando a aderência ao tratamento.

Os ACC não apresentam qualquer efeito desfavorável sobre o metabolismo glicídio, não interferem na resistência insulínica, nem modificam o perfil lipídico.

Quanto aos efeitos cardíacos, os ACC são bastante expressivos; a insuficiência coronariana pode estar presente em muitos hipertensos e o emprego dos ACC pode ser útil para tratar ambas as situações, reduzindo significativamente o número de episódios anginosos. No que se refere aos efeitos sobre a progressão da doença ateroesclerótica, os dados disponíveis ainda são pouco consistentes, havendo apenas resultados importantes em modelos animais. Os estudos clínicos sobre redução ou desaceleração de doença ateroesclerótica de carótida têm sido pouco animadores.

Por outro lado, estudos analisando a regressão de hipertrofia ventricular esquerda têm sido mais promissores, observando-se a redução da espessura da parede livre do ventrículo esquerdo e das dimensões diastólicas finais, sem mudanças nos índices de enchimento diastólico, além de promoverem melhora na complacência ventricular, com repercussões benéficas na função diastólica do ventrículo esquerdo. Os ACC não interferem nas respostas cardiovasculares durante atividades físicas.

Há evidências clínicas e experimentais de que os ACC diminuem a proteinúria em hipertensos diabéticos, além de provavelmente determinarem uma redução na velocidade de perda da função renal. Estes efeitos protetores renais não devem ser mediados, primariamente, por alterações na hemodinâmica intra-renal mas, provavelmente, por alterações antitróficas destas drogas.

Os ACC apresentam poucas contra-indicações. Deve-se evitar a associação do verapamil com qualquer beta bloqueador na presença de bradicardia importante ou evidências clínicas de insuficiência cardíaca. Da mesma maneira não devem ser prescrito, imediatamente, após um acidente coronariano agudo, principalmente na presença de insuficiência cardíaca. Única exceção se faz ao diltiazem nos infartos agudo do miocárdio sem onda Q. 
Os efeitos colaterais observados são específicos de cada grupo: os dihidropirídinicos podem desencadear cefaléias importantes que muitas das vezes obrigam os pacientes a suspender a medicação. $\mathrm{O}$ "flushing" facial é outro efeito adverso, menos comum que a cefaléia, mas que também leva os pacientes a abandonar o tratamento. A taquicardia é aparentemente mais suportável e, por vezes, é necessário a associação de um beta bloqueador para minimizar este efeito. O edema pedal, decorrente de alterações hemodinâmicas locais, não é reduzido pelo uso concomitante de diuréticos, sendo outro efeito que pode levar à suspensão do tratamento. A hiperplasia gengival é um efeito colateral raro que o médico deve lembrar da associação quando referido pelo paciente.

A constipação intestinal é o efeito colateral mais freqüente com o uso de verapamil. Deve-se tomar cuidado quando do uso em pacientes portadores de distúrbios no sistema de condução cardíaco e, também, no uso concomitante de digoxina uma vez que o verapamil aumenta seus níveis séricos.

O diltiazem promove menos efeitos gastrointestinais quando comparados ao verapamil e, também deve-se evitá-lo em pacientes com distúrbio nos sistema de condução cardíaco.

\subsection{Inibidores da Enzima de Conversão}

O sistema renina-angiotensina (SRA) desempenha um papel fundamental na homeostase circulatória. Em situações agudas de perda de volume ou queda súbita da pressão arterial, observa-se uma rápida ativação do sistema. Por outro lado, quando se promove expansão volumétrica observa-se redução da atividade do SRA.

Na hipertensão arterial humana, o papel do SRA na sua fisiopatologia ainda não está definitivamente esclarecido. Porém, a interrupção farmacológica deste sistema leva ao controle da pressão arterial de muitos pacientes, além de reverter uma série de alterações estruturais como a hipertrofia miocárdica e vascular.

Ao se cotejar a excreção urinária de sódio, nas 24 horas, versus a atividade plasmática de renina pode-se identificar 3 subgrupos de hipertensos: aqueles com renina alta (10 a 20\%); renina baixa ( 15 a $25 \%$ ) e o terceiro denominado de renina normal (50 a $65 \%)$ ou "inapropriadamente" normal, uma vez que estando a pressão elevada deveria o sistema estar inativado. Dessa forma, cerca de $60 \%$ dos hipertensos podem se beneficiar dos inibidores da ECA. Contudo, na prática diária não é necessário medir-se a atividade de renina plasmática para prescrever um inibidor da ECA. A identificação do sistema renina-angiotensina ao nível tecidual veio explicar por que alguns hipertensos mesmo tendo atividade do SRA, no plasma, reduzida respondem bem aos inibidores do ECA.

A introdução dos inibidores da ECA no arsenal terapêutico para HA representa um avanço quanto à especificidade na abordagem do tratamento $\mathrm{e}$, assim, menos efeitos colaterais.

Sob ponto de vista hemodinâmico, diminuem a resistência vascular periférica sem interferirem no débito cardíaco, além de preservarem os fluxos regionais principalmente cerebral, coronariano e renal.

Os inibidores da ECA não interferem no metabolismo lipídico; quanto ao metabolismo glicídio há evidências experimentais que levam a uma maior sensibilidade periférica à insulina, com redução nos seus níveis plasmáticos sendo uma das drogas de indicação preferencial para diabéticos. Estes efeitos são, provavelmente, mediados por um aumento local de bradicinina.

$\mathrm{O}$ efeito dos inibidores da ECA promovem regressão da hipertrofia ventricular esquerda em graus maiores que as demais drogas anti-hipertensivas. Estão, também, indicados para os hipertensos com insuficiência cardíaca e atenuam a progressão da dilatação ventricular esquerda em pacientes com baixa fração de ejeção com ou sem infarto do miocárdio recente.

No rim, os inibidores da ECA interferem com a hemodinâmica local ao promoverem dilatação preferencial da arteríola eferente, levando à redução na hipertensão intraglomerular. Estas alterações hemodinâmicas determinam melhora do fluxo, redução na progressão da agressão glomerular, diminuição na velocidade de perda da função, principalmente, em pacientes com proteinúria por diabetes. Este efeito é, também, observado em pacientes diabéticos normotensos com proteinúria.

Em geral, os inibidores da ECA podem ser administrados uma vez ao dia. Na necessidade de se aumentar seu efeito hipotensor pode-se optar por aumentar a sua dose e administrá-la 2 vezes ao dia ou, então, manter a dose inicial e associar um diurético tiazídicos em baixas doses de (12,5 mg). Em pacientes que já venham em uso de diurético, pode se observar uma queda súbita da pressão arterial com hipotensão sintomática. Esse efeito é mais freqüentemente observado em pacientes quando se administra o captopril, uma vez que esse inibidor da ECA, em contraste aos demais, é administrado na sua forma ativa, 
é de ação mais rápida não sendo necessária sua metabolização pelo fígado.

Dos efeitos colaterais, a tosse seca representa o evento mais freqüente relacionado à família dos inibidores da ECA, i.e., não adianta substituir por outro inibidor que a "chance" da tosse voltar é muito grande. Os demais efeitos, como distúrbio no paladar, rash cutâneo, leucopenia, estão mais relacionados à estrutura química dos inibidores da ECA que apresentam radical sulfidrila na sua molécula e estas alterações são mais freqüentes quando administrados em doses elevadas. Em geral, estes efeitos são revertidos com a suspensão da medicação.

Os inibidores da ECA estão, formalmente, contra-indicados na gravidez. É provável que sejam excretados pelo leito materno, e mulheres, em fase fértil, devem suspendê-lo na suspeita de gravidez.

\subsection{Antagonistas do receptor da Angiotensina II}

A saralasina foi o primeiro agonista da angiotensina II a ser empregada na clínica com boa atividade anti-hipertensiva. Apresentava, porém, alguns problemas práticos: não podia ser administrado por via oral por ser um peptídio e, apresentava em alguns hipertensos uma atividade agonista parcial, ou seja, ao invés de abaixar a pressão ela a elevava, principalmente em hipertensos com atividade muito reduzida da renina plasmática.

Mas recentemente, foram sintetizados agonistas específicos de receptores da Angiotensina II, alguns deles como o Losartan, com grande experiência clínica. Estes antagonistas são denominados de específicos porque bloqueiam apenas o receptor $\mathrm{At}_{1}$, deixando livre o receptor $\mathrm{At}_{2}$.

No presente momento, já estão bem reconhecidas algumas das funções destes receptores. Por exem- plo, o receptor $\mathrm{At}_{1}$ é responsável por diversas ações biológicas, incluindo a vasoconstrição, a secreção e liberação adrenal de aldosterona. A estimulação destes receptores desencadeia uma série de eventos bioquímicos em cascata ao nível intracelular, envolvendo o PI (fosfato de inositol), a geração de segundos mensageiros como o DAG (diacilglicerol) que, por sua vez, aumenta a quantidade cálcio intracelular. Esse aumento da concentração de cálcio na musculatura lisa dos vasos e do miocárdio determina vasoconstrição e, também, aumento do inotropismo além de levar a aumento na liberação de catecolaminas e neurotransmissores. Essas alterações bioquímicas explicam os outros efeitos da AII que podem resultar em alterações estruturais nos vasos e miocárdio.

As funções do receptor $\mathrm{At}_{2}$ não estão claras quanto àquelas mediadas pelos receptores $\mathrm{At}_{1}$. Há evidências experimentais que apontam este receptor, $\mathrm{At}_{2}$, como um modulador de atividades tróficas mediadas pela AII. Os resultados mostram que o $\mathrm{At}_{2}$ reduziria os estímulos pró-sintese do $\mathrm{At}_{1}$, ou seja, teria uma ação de regular a proliferação estimulada pela AII. Sob ponto de vista terapêutico, não parece haver qualquer sentido empregar uma droga que antagonize este receptor.

O antagonista de AII, losartan, é administrado na sua forma ativa e o seu metabólito, também, apresenta atividade anti-hipertensiva, o que confere um efeito prolongado da sua ação podendo, desta maneira, ser administrado uma vez ao dia. A eficácia anti-hipertensiva e seus efeitos sobre o coração e sobre o rim parecem ser semelhantes aos dos inibidores da enzima de conversão. Contudo, seus efeitos adversos são bem diferentes, principalmente com relação à tosse, cuja incidência é, significativamente, menor comparado aos inibidores da ECA.

OIGMAN W. Pharmacology treatment of arterial hypertension. Medicina, Ribeirão Preto, 29: 244-249, apr./sept. 1996.

ABSTRACT: This article reviews objectively the 7 principals antihypertensive families used in clinical practice. The author enforced the individualized treatment pointing for specifically indications when other diseases or cardiovascular risk factor being present. The hemodinamic and metabolic repercussions of each antihypertensive group are presented in detail.

UNITERMS: Hypertension; pharmacology. Antihypertensive Agents. 


\section{BIBLIOGRAFIA RECOMENDADA}

1 - BRUNNER HR; MENARD $J$ \& WAEBER B. Treating the individual hypertensive patient: considerations on dose, sequential monotherapy and drug combinations. J Hypertens 8:3-11, 1990.

2 - II CONSENSO BRASILEIRO DE HIPERTENSÃO ARTERIAL. J Bras Nefrol 16 (Supl 2): S 257-278, 1994.

3 - FLECKENSTEIN A. History and prospects in calcium antagonist research. J Mol Cell Cardiol 22:241-251, 1990

4 - GOLDEBERG AI; DUNLAY MC \& SWEET CS. Safety and tolerability of losartan potassium, an angiotensin II receptor antagonist, compared with hydrochlorothiazide, atenolol, felodipine ER, and angiotensin_converting enzyme Inhibitors for the treatment of systemic hypertension. Am J Cardiol 75:793-795, 1995.

5 - HANSSON L \& HIMMELMANN A. Calcium antagonists in antihypertensive combination therapy. J Cardiovasc Pharmacol 18 (Suppl 10) :S76-80, 1991.

6 - JAMES MA \& JONES JV. An interaction between LVH and potassium in hypertension? J Hum Hypertens 5:475-478, 1991.

7 - THE FIFTH REPORT JOINT NATIONAL COMMITTEE ON DETECTION EVALU-ATION AND TREATMENT OF HIGH BLOOD PRESSURE. Arch Intern Med 153: 154-183, 1993.

8 - MACMAHON S; PETO R \& CUTLER J. Blood pressure, stroke, and coronary heart disease. Part 1, Prolonged differences in blood opressure: prospective observational studies corrected for the regression dilution bias. Lancet 335:765-774, 1990.

9 - OIGMAN W. Combinação de drogas para a hipertensão arterial. Algumas considerações práticas. Rev Bras Med; 43:219-223, 1989.
10 - OPIE LH. Calcium channel antagonists Part I: Fundamental properties: mechanisms, classification, sites of action. Cardiovasc Drugs Ther 1: 411-430, 1987.

11 - van SCHAIK BAM, GEYSKES GG \& MEES EJD. The effects of converting enzime inhibition on the enhanced proximal sodium reabsorption induced by chronic diuretic treatment in patients with essential hypertension. Nephron 47: 167-172, 1987.

12 - SHEP COOPERATIVE RESEARCH GROUP. Prevention of stroke by antihypertensive drug treatment in older persons with isolated systolic hypertension. Final results of the Systolic Hypertension in the elderly program. JAMA 265:3255-3264, 1992.

13 - WARRAM JH et al. Excess mortality associated with diuretic therapy in diabetis mellitus. Arch Intern Med 151 1350-1356, 1991

14 - WEBER MA et al. Blood pressure effects of the angiotensin II receptor Blocker, Losartan. Arch Intern Med 155 405-411, 1995.

15 - WILLIAMS GH. Converting enzyme inhibitors in the treatment of hypertension. N Engl J Med 319:1517-1525, 1988.

16 - van ZWIETEN PA; THOOLEN MJMC \& TIMMERMANS PBM. The hypontesive activity and side effects of methyldopa, clonidine, and guanfacine. Hypertension 6 (suppl 2): S-28-33,1984.

Recebido para publicação em 16/01/96

Aprovado para publicação em 25/04/96 